\title{
On Approximation of Analytic Functions by Periodic Hurwitz Zeta-Functions
}

\section{Violeta Franckevič ${ }^{a}$, Antanas Laurinčikas ${ }^{a}$ and Darius Šiaučiūnas ${ }^{b, c}$}

${ }^{a}$ Institute of Mathematics, Faculty of Mathematics and Informatics, Vilnius University

Naugarduko str. 24, LT-03225 Vilnius, Lithuania

${ }^{b}$ Department of Computer Sciences, Siauliai University

Vilniaus str. 141, LT-76353 Šiauliai, Lithuania

${ }^{c}$ Research Institute, Šiauliai University

P. Višinskio str. 25, LT-76351 Šiauliai, Lithuania

E-mail(corresp.): darius.siauciunas@su.lt

E-mail: violeta.franckevic@stud.mif.vu.lt

E-mail: antanas.laurincikas@mif.vu.lt

Received June 13, 2018; revised October 10, 2018; accepted October 12, 2018

\begin{abstract}
The periodic Hurwitz zeta-function $\zeta(s, \alpha ; \mathfrak{a}), s=\sigma+i t$, with parameter $0<\alpha \leqslant 1$ and periodic sequence of complex numbers $\mathfrak{a}=\left\{a_{m}\right\}$ is defined, for $\sigma>1$, by the series $\sum_{m=0}^{\infty} \frac{a_{m}}{(m+\alpha)^{s}}$, and can be continued moromorphically to the whole complex plane. It is known that the function $\zeta(s, \alpha ; \mathfrak{a})$ with transcendental or rational $\alpha$ is universal, i.e., its shifts $\zeta(s+i \tau, \alpha ; \mathfrak{a})$ approximate all analytic functions defined in the strip $D=\left\{s \in \mathbb{C}: \frac{1}{2}<\sigma<1\right\}$. In the paper, it is proved that, for all $0<\alpha \leqslant 1$ and $\mathfrak{a}$, there exists a non-empty closed set $F_{\alpha, \mathfrak{a}}$ of analytic functions on $D$ such that every function $f \in F_{\alpha, \mathfrak{a}}$ can be approximated by shifts $\zeta(s+i \tau, \alpha ; \mathfrak{a})$.
\end{abstract}

Keywords: Hurwitz zeta-function, periodic Hurwitz zeta-function, universality, weak convergence of probability measures.

AMS Subject Classification: 11M41; 11M35.

\section{Introduction}

Let $\alpha, 0<\alpha \leqslant 1$, be a fixed parameter, and let $\mathfrak{a}=\left\{a_{m} \in \mathbb{C}: m \in \mathbb{N}_{0}\right\}$, $\mathbb{N}_{0}=\mathbb{N} \cup\{0\}$, be a periodic sequence with minimal period $q \in \mathbb{N}$. The periodic

Copyright (c) 2019 The Author(s). Published by VGTU Press

This is an Open Access article distributed under the terms of the Creative Commons Attribution License (http://creativecommons.org/licenses/by/4.0/), which permits unrestricted use, distribution, and reproduction in any medium, provided the original author and source are credited. 
Hurwitz zeta-function $\zeta(s, \alpha ; \mathfrak{a}), s=\sigma+i t$, is defined, for $\sigma>1$, by the Dirichlet series

$$
\zeta(s, \alpha ; \mathfrak{a})=\sum_{m=0}^{\infty} \frac{a_{m}}{(m+\alpha)^{s}} .
$$

If $a_{m} \equiv 1$, then $\zeta(s, \alpha ; \mathfrak{a})$ reduces to the classical Hurwitz zeta-function

$$
\zeta(s, \alpha)=\sum_{m=0}^{\infty} \frac{1}{(m+\alpha)^{s}}, \quad \sigma>1,
$$

which is analytically continued to the whole complex plane, except for the point $s=1$ that is a simple pole with residue 1 . The periodicity of the sequence $\mathfrak{a}$ implies the equality

$$
\zeta(s, \alpha ; \mathfrak{a})=\frac{1}{q^{s}} \sum_{l=0}^{q-1} a_{l} \zeta\left(s, \frac{l+\alpha}{q}\right), \quad \sigma>1 .
$$

Therefore, the periodic Hurwitz zeta-function, as $\zeta(s, \alpha)$, has analytic continuation to the whole complex plane, except for the point $s=1$ that is a simple pole with residue

$$
\frac{1}{q} \sum_{l=0}^{q-1} a_{l}
$$

If $a_{0}+\cdots+a_{q-1}=0$, then the function $\zeta(s, \alpha ; \mathfrak{a})$ is entire.

The function $\zeta(s, \alpha ; \mathfrak{a})$ is also connected with the Lerch zeta-function $L(\lambda$, $\alpha, s), \lambda \in \mathbb{R}$, which, for $\sigma>1$, is given by the series

$$
L(\lambda, \alpha, s)=\sum_{m=0}^{\infty} \frac{\mathrm{e}^{2 \pi i \lambda m}}{(m+\alpha)^{s}} .
$$

For rational $\lambda$, the function $L(\lambda, \alpha, s)$ becomes the periodic Hurwitz zetafunction. Thus, the function $\zeta(s, \alpha ; \mathfrak{a})$ is an extension of the Lerch zeta-function with rational parameter $\lambda$.

The above remarks show that the periodic Hurwitz zeta-function is a generalization of the classical Hurwitz and Lerch zeta-functions.

It is well known that some zeta-functions are universal in the Voronin sense who discovered [9] the universality of the Riemann zeta-function $\zeta(s)$. More precisely, the modern version of the Voronin theorem, see, for example, [4,8], states that if $D=\left\{s \in \mathbb{C}: \frac{1}{2}<\sigma<1\right\}, \mathcal{K}$ is the class of compact subsets of the strip $D$ with connected complements, and $H_{0}(K)$ with $K \in \mathcal{K}$ is the class of continuous non-vanishing functions on $K$ that are analytic in the interior of $K$, then, for $K \in \mathcal{K}, f(s) \in H_{0}(K)$ and every $\varepsilon>0$,

$$
\liminf _{T \rightarrow \infty} \frac{1}{T} \operatorname{meas}\left\{\tau \in[0, T]: \sup _{s \in K}|\zeta(s+i \tau)-f(s)|<\varepsilon\right\}>0 .
$$

Here meas $A$ denotes the Lebesgue measure of a measurable set $A \subset \mathbb{R}$. The above inequality shows that the set of shifts $\zeta(s+i \tau)$ approximating uniformly 
on $K \in \mathcal{K}$ a given function $f(s) \in H_{0}(K)$ with accuracy $\varepsilon$ is infinite because it has a positive lower density.

Universality of zeta-functions is one of the most interesting phenomenons of analytic number theory and has numerous theoretical and practical applications. Therefore, after Voronin's work, various authors continued the investigations of universality of zeta-functions and obtained significant results. The results, methods, problems and references on the universality of zeta-functions can be found in an excellent survey paper [6].

Now we return to the periodic Hurwitz zeta-function. The function $\zeta(s, \alpha ; \mathfrak{a})$ depends on the parameter $\alpha$ and periodic sequence $\mathfrak{a}$, therefore, its valuedistribution, including the universality, is influenced by properties of the latter objects. The parameter $\alpha$ can be transcendental, rational or algebraic irrational. The case of transcendental $\alpha$ is the simplest one, and the universality of $\zeta(s, \alpha ; \mathfrak{a})$ in this case has been proved in [3]. Let $H(K)$ with $K \in \mathcal{K}$ denote the class of continuous functions on $K$ that are analytic in the interior of $K$. Thus, we have that $H_{0}(K) \subset H(K)$. Then the main result of [3] is the following theorem.

Theorem 1. Suppose that the parameter $\alpha$ is transcendental. Let $K \in \mathcal{K}$ and $f(s) \in H(K)$. Then, for every $\varepsilon>0$,

$$
\liminf _{T \rightarrow \infty} \frac{1}{T} \operatorname{meas}\left\{\tau \in[0, T]: \sup _{s \in K}|\zeta(s+i \tau, \alpha ; \mathfrak{a})-f(s)|<\varepsilon\right\}>0 .
$$

The universality of the function $\zeta(s, \alpha ; \mathfrak{a})$ with rational $\alpha$ has been studied in [5], and the following result was obtained. We remind that $q$ is the period of the sequence $\mathfrak{a}$.

Theorem 2. Suppose that $\alpha=\frac{a}{b}, a, b \in \mathbb{N}, a<b,(a, b)=1, \alpha \neq \frac{1}{2}$ and $(b l+a, b q)=1$ for all $l=0,1, \ldots, q-1$. Let $K \in \mathcal{K}$ and $f(s) \in H(K)$. Then the assertion of Theorem 1 is valid.

The universality of $\zeta(s, \alpha ; \mathfrak{a})$ with algebraic irrational parameter $\alpha$ remains an open problem until our days. Therefore, in this paper, we propose the following "approximation" to the universality of the function $\zeta(s, \alpha ; \mathfrak{a})$. We state the theorems for all parameters $\alpha$ and sequences $\mathfrak{a}$ because there are a bit different from universality theorems with transcendental or rational parameter. Denote by $H(D)$ the space of analytic functions on $D$ endowed with the topology of uniform convergence on compacta.

Theorem 3. Suppose that the parameter $\alpha, 0<\alpha \leqslant 1$, and the periodic sequence $\mathfrak{a}$ are arbitrary. Then there exists a non-empty closed set $F_{\alpha, \mathfrak{a}} \subset H(D)$ such that, for every compact subset $K \subset D, f(s) \in F_{\alpha, \mathfrak{a}}$ and $\varepsilon>0$,

$$
\liminf _{T \rightarrow \infty} \frac{1}{T} \operatorname{meas}\left\{\tau \in[0, T]: \sup _{s \in K}|\zeta(s+i \tau, \alpha ; \mathfrak{a})-f(s)|<\varepsilon\right\}>0 .
$$

The positivity of a lower density of the set of $\operatorname{shifts} \zeta(s+i \tau, \alpha ; \mathfrak{a})$ can be replaced by the positivity of the density of that set. More precisely, we have the following modification of Theorem 3 . 
Theorem 4. Suppose that the parameter $\alpha, 0<\alpha \leqslant 1$, and the periodic sequence $\mathfrak{a}$ are arbitrary. Then there exists a non-empty closed set $F_{\alpha, \mathfrak{a}} \subset H(D)$ such that, for every compact subset $K \subset D$ and $f(s) \in F_{\alpha, \mathfrak{a}}$, the limit

$$
\lim _{T \rightarrow \infty} \frac{1}{T} \operatorname{meas}\left\{\tau \in[0, T]: \sup _{s \in K}|\zeta(s+i \tau, \alpha ; \mathfrak{a})-f(s)|<\varepsilon\right\}>0
$$

exists for all but at most countably many $\varepsilon>0$.

Unfortunately, the set $F_{\alpha, \mathfrak{a}}$ is not explicitly given. We will prove, that this set is the support of a certain probability measure.

Theorems 3 and 4 can be generalized for some compositions. We give one example.

Theorem 5. Suppose that the parameter $\alpha, 0<\alpha \leqslant 1$, and the periodic sequence $\mathfrak{a}$ are arbitrary. Then there exists a non-empty closed set $F_{\alpha, \mathfrak{a}} \subset H(D)$ such that if $\Phi: H(D) \rightarrow H(D)$ is a continuous operator such that, for every polynomial $p=p(s)$, the set $\left(\Phi^{-1}\{p\}\right) \cap F_{\alpha, \mathfrak{a}}$ is non-empty, then, for every compact subset $K \subset D, f(s) \in \Phi\left(F_{\alpha, \mathfrak{a}}\right)$ and $\varepsilon>0$,

$$
\liminf _{T \rightarrow \infty} \frac{1}{T} \operatorname{meas}\left\{\tau \in[0, T]: \sup _{s \in K}|\Phi(\zeta(s+i \tau, \alpha ; \mathfrak{a}))-f(s)|<\varepsilon\right\}>0 .
$$

The next theorem is an analogue of Theorem 4 for the composition $\Phi(\zeta(s$ $+i \tau, \alpha ; \mathfrak{a}))$.

Theorem 6. Suppose that the parameter $\alpha, 0<\alpha \leqslant 1$, and the periodic sequence $\mathfrak{a}$ are arbitrary. Then there exists a non-empty closed set $F_{\alpha, \mathfrak{a}} \subset H(D)$ such that if $\Phi: H(D) \rightarrow H(D)$ is a continuous operator such that, for every polynomial $p=p(s)$, the set $\left(\Phi^{-1}\{p\}\right) \cap F_{\alpha, \mathfrak{a}}$ is non-empty, then, for every compact subset $K \subset D$ and $f(s) \in \Phi\left(F_{\alpha, \mathfrak{a}}\right)$, the limit

$$
\lim _{T \rightarrow \infty} \frac{1}{T} \operatorname{meas}\left\{\tau \in[0, T]: \sup _{s \in K}|\Phi(\zeta(s+i \tau, \alpha ; \mathfrak{a}))-f(s)|<\varepsilon\right\}>0
$$

exists for all but at most countably many $\varepsilon>0$.

For the proof of above theorems, we apply a probabilistic approach based on limit theorems for weakly convergent probability measures in the space $H(D)$. These limit theorems will be proved in the next section.

\section{Limit theorems}

Denote by $\mathcal{B}(X)$ the Borel $\sigma$-field of the space $X$. In this section, we will prove a limit theorem for

$$
P_{T, \alpha, \mathfrak{a}}(A)=\frac{1}{T} \operatorname{meas}\{\tau \in[0, T]: \zeta(s+i \tau, \alpha ; \mathfrak{a}) \in A\}, \quad A \in \mathcal{B}(H(D)),
$$

as $T \rightarrow \infty$. 
Theorem 7. Suppose that the parameter $\alpha, 0<\alpha \leqslant 1$, and the periodic sequence $\mathfrak{a}$ are arbitrary. Then, on $(H(D), \mathcal{B}(H(D)))$, there exists a probability measure $P_{\alpha, \mathfrak{a}}$ such that $P_{T, \alpha, \mathfrak{a}}$ converges weakly to $P_{\alpha, \mathfrak{a}}$ as $T \rightarrow \infty$.

First, we will prove a limit theorem for probability measures on $(\Omega, \mathcal{B}(\Omega))$, where $\Omega=\prod_{m=0}^{\infty} \gamma_{m}$, and $\gamma_{m}=\{s \in \mathbb{C}:|s|=1\}$ for all non-negative integers $m$. Since the unit circle is a compact set, by the Tikhonov theorem, the infinitedimensional torus $\Omega$ with the product topology and pointwise multiplication is a compact topological Abelian group. Denote by $\omega(m), m \in \mathbb{N}_{0}$, the $m$-th component of an element $\omega \in \Omega$. Clearly, $\left((m+\alpha)^{-i \tau}: m \in \mathbb{N}_{0}\right)$ is an element of $\Omega$. For $A \in(\Omega, \mathcal{B}(\Omega))$, define

$$
Q_{T, \alpha}(A)=\frac{1}{T} \text { meas }\left\{\tau \in[0, T]:\left((m+\alpha)^{-i \tau}: m \in \mathbb{N}_{0}\right) \in A\right\} .
$$

In the sequel, we assume that the parameter $\alpha, 0<\alpha \leqslant 1$, and the sequence $\mathfrak{a}$ are arbitrary.

Lemma 1. On $(\Omega, \mathcal{B}(\Omega))$, there exists a probability measure $Q_{\alpha}$ such that $Q_{T, \alpha}$ converges weakly to $Q_{\alpha}$ as $T \rightarrow \infty$.

Proof. The dual group of $\Omega$ is isomorphic to $\bigoplus_{m=0}^{\infty} \mathbb{Z}_{m}$, where $\mathbb{Z}_{m}=\mathbb{Z}$ for all $m \in \mathbb{N}$. Therefore, the characters $\chi$ of the group $\Omega$ are of the form

$$
\chi(m)=\prod_{m=0}^{\infty} \omega^{k_{m}}(m), \quad \omega \in \Omega,
$$

where only a finite number of integers $k_{m}$ are distinct from zero. Hence, the Fourier transform $g_{T, \alpha, \mathfrak{a}}(\underline{k}), \underline{k}=\left(k_{m} \in \mathbb{Z}: m \in \mathbb{N}_{0}\right)$, of $Q_{T, \alpha}$ is defined by

$$
g_{T, \alpha}(\underline{k})=\int_{\Omega}\left(\prod_{m=0}^{\infty} \omega^{k_{m}(m)}\right) \mathrm{d} Q_{T, \alpha},
$$

where only a finite number of integers $k_{m}$ are distinct from zero. We consider $g_{T, \alpha}$ as $T \rightarrow \infty$. Define two collections

$$
\begin{aligned}
& \underline{k}_{1, \alpha}=\left\{\underline{k}=\left\{k_{m} \in \mathbb{Z}\right\}: \sum_{m=0}^{\infty} k_{m} \log (m+\alpha)=0\right\}, \\
& \underline{k}_{2, \alpha}=\left\{\underline{k}=\left\{k_{m} \in \mathbb{Z}\right\}: \sum_{m=0}^{\infty} k_{m} \log (m+\alpha) \neq 0\right\},
\end{aligned}
$$

where the sign " 1 " means that only a finite number of integers $k_{m}$ are distinct from zero. In view of (2.1) and the definition of $Q_{T, \alpha}$,

$$
\begin{aligned}
g_{T, \alpha}(\underline{k}) & =\frac{1}{T} \int_{0}^{T}\left(\prod_{m=0}^{\infty}(m+\alpha)^{-i \tau k_{m}}\right) \mathrm{d} \tau \\
& =\frac{1}{T} \int_{0}^{T} \exp \left\{-i \tau \sum_{m=0}^{\infty} k_{m} \log (m+\alpha)\right\} \mathrm{d} \tau .
\end{aligned}
$$


Obviously,

$$
g_{T, \alpha}(\underline{k})=1
$$

for $\underline{k} \in\left\{\underline{k}_{1, \alpha}\right\}$. If $\underline{k} \in\left\{\underline{k}_{2, \alpha}\right\}$, then after integration we have that

$$
g_{T, \alpha}(\underline{k})=\frac{1-\exp \left\{-i T \sum_{m=0}^{\prime \infty} k_{m} \log (m+\alpha)\right\}}{i T \sum_{m=0}^{\infty} k_{m} \log (m+\alpha)} .
$$

Therefore, in this case,

$$
\lim _{T \rightarrow \infty} g_{T, \alpha}(\underline{k})=0 .
$$

This and (2.2) show that

$$
\lim _{T \rightarrow \infty} g_{T, \alpha}(\underline{k})=\left\{\begin{array}{lll}
1 & \text { if } & \underline{k} \in\left\{\underline{k}_{1, \alpha}\right\}, \\
0 & \text { if } & \underline{k} \in\left\{\underline{k}_{2, \alpha}\right\} .
\end{array}\right.
$$

Therefore, we obtained that the Fourier transform of $Q_{T, \alpha}$, as $T \rightarrow \infty$, converges to a continuous function in the discrete topology. Thus, by a continuity theorem for probability measures on compact groups, we deduce that $Q_{T, \alpha}$, as $T \rightarrow \infty$, converges weakly to a probability measure $Q_{\alpha}$ on $(\Omega, \mathcal{B}(\Omega))$ given by the Fourier transform

$$
g_{\alpha}(\underline{k})=\left\{\begin{array}{lll}
1 & \text { if } & \underline{k} \in\left\{\underline{k}_{1, \alpha}\right\}, \\
0 & \text { if } \quad \underline{k} \in\left\{\underline{k}_{2, \alpha}\right\} .
\end{array}\right.
$$

Lemma 1 is applied for proving a limit theorem for absolutely convergent Dirichlet series. Let $\theta>\frac{1}{2}$ be a fixed number. Define

$$
\begin{aligned}
& v_{n}(m, \alpha)=\exp \left\{-\left(\frac{m+\alpha}{n+\alpha}\right)^{\theta}\right\}, \quad m \in \mathbb{N}_{0}, n \in \mathbb{N}, \\
& \zeta_{n}(s, \alpha, \mathfrak{a})=\sum_{m=0}^{\infty} \frac{a_{m} v_{n}(m, \alpha)}{(m+\alpha)^{s}} .
\end{aligned}
$$

Then it is known [2] that the latter series is absolutely convergent for $\sigma>\frac{1}{2}$. For $A \in \mathcal{B}(H(D))$, let

$$
P_{T, n, \alpha, \mathfrak{a}}(A)=\frac{1}{T} \text { meas }\left\{\tau \in[0, T]: \zeta_{n}(s+i \tau, \alpha, \mathfrak{a}) \in A\right\} .
$$

Lemma 2. On $(H(D), \mathcal{B}(H(D)))$, there exists a probability measure $V_{n, \alpha, \mathfrak{a}}$ such that $P_{T, n, \alpha, \mathfrak{a}}$ converges weakly to $V_{n, \alpha, \mathfrak{a}}$ as $T \rightarrow \infty$.

Proof. Consider the function $u_{n, \alpha, \mathfrak{a}}: \Omega \rightarrow H(D)$ given by

$$
u_{n, \alpha, \mathfrak{a}}(\omega)=\sum_{m=0}^{\infty} \frac{a_{m} \omega(m) v_{n}(m, \alpha)}{(m+\alpha)^{s}}, \quad \omega \in \Omega .
$$


Since $|\omega(m)|=1$, the latter series, as the series for $\zeta_{n}(s, \alpha, \mathfrak{a})$, is absolutely convergent for $\sigma>\frac{1}{2}$. Hence, the function $u_{n, \alpha, \mathfrak{a}}$ is continuous, therefore, it is $(\mathcal{B}(\Omega), \mathcal{B}(H(D)))$-measurable. Thus, the limit measure $Q_{\alpha}$ of Lemma 1 induces on $(H(D), \mathcal{B}(H(D)))$ the unique probability measure $V_{n, \alpha, \mathfrak{a}} \stackrel{\text { def }}{=} Q_{\alpha} u_{n, \alpha, \mathfrak{a}}^{-1}$, where

$$
Q_{\alpha} u_{n, \alpha, \mathfrak{a}}^{-1}(A)=Q_{\alpha}\left(u_{n, \alpha, \mathfrak{a}}^{-1} A\right), \quad A \in \mathcal{B}(H(D)) .
$$

Clearly,

$$
u_{n, \alpha, \mathfrak{a}}\left((m+\alpha)^{-i \tau}: m \in \mathbb{N}_{0}\right)=\sum_{m=0}^{\infty} \frac{a_{m} v_{n}(m, \alpha)}{(m+\alpha)^{s+i \tau}}
$$

Therefore,

$$
\begin{aligned}
P_{T, n, \alpha, \mathfrak{a}}(A) & =\frac{1}{T} \operatorname{meas}\left\{\tau \in[0, T]:\left((m+\alpha)^{-i \tau}: m \in \mathbb{N}_{0}\right) \in u_{n, \alpha, \mathfrak{a}}^{-1} A\right\} \\
& =Q_{T, \alpha}\left(u_{n, \alpha, \mathfrak{a}}^{-1} A\right)=Q_{T, \alpha} u_{n, \alpha, \mathfrak{a}}^{-1}(A)
\end{aligned}
$$

This shows that $P_{T, n, \alpha, \mathfrak{a}}=Q_{T, \alpha} u_{n, \alpha, \mathfrak{a}}^{-1}$. Since the function $u_{n, \alpha, \mathfrak{a}}$ is continuous, and, by Lemma $1, Q_{T, \alpha}$, as $T \rightarrow \infty$, converges weakly to $Q_{\alpha}$, we obtain by Theorem 5.1 of [1] that $P_{T, n, \alpha, \mathfrak{a}}$ converges weakly to $V_{n, \alpha, \mathfrak{a}}=Q_{\alpha} u_{n, \alpha, \mathfrak{a}}^{-1}$ as $T \rightarrow \infty$.

Now, we will approximate in the mean the function $\zeta(s, \alpha ; \mathfrak{a})$ by $\zeta_{n}(s, \alpha ; \mathfrak{a})$. Denote by $\rho$ the metric in $H(D)$ inducing the topology of uniform convergence on compacta.

\section{Lemma 3. The equality}

$$
\lim _{n \rightarrow \infty} \limsup _{T \rightarrow \infty} \frac{1}{T} \int_{0}^{T} \rho\left(\zeta(s+i \tau, \alpha ; \mathfrak{a}), \zeta_{n}(s+i \tau, \alpha ; \mathfrak{a})\right) \mathrm{d} \tau=0
$$

holds.

Proof. Let the number $\theta$ be from the definition of the function $v_{n}(m, \alpha)$. Then it is known [2] that, for $\sigma>\frac{1}{2}$,

$$
\zeta_{n}(s, \alpha ; \mathfrak{a})=\frac{1}{2 \pi i} \int_{\theta-i \infty}^{\theta+i \infty} \zeta(s+z, \alpha ; \mathfrak{a}) l_{n}(z, \alpha) \frac{\mathrm{d} z}{z},
$$

where

$$
l_{n}(s, \alpha)=\frac{s}{\theta} \Gamma\left(\frac{s}{\theta}\right)(n+\alpha)^{s},
$$

and $\Gamma(s)$ denotes the Euler gamma-function. For an arbitrary $\beta>0$, the residue theorem and $(2.3)$ give

$$
\begin{aligned}
\zeta_{n}(s, \alpha ; \mathfrak{a})-\zeta(s, \alpha ; \mathfrak{a})= & \frac{1}{2 \pi i} \int_{-\beta-i \infty}^{-\beta+i \infty} \zeta(s+z, \alpha ; \mathfrak{a}) l_{n}(z, \alpha) \frac{\mathrm{d} z}{z} \\
& +\operatorname{Res}_{z=1-s} \frac{l_{n}(z, \alpha)}{z} \zeta(s+z, \alpha ; \mathfrak{a}) .
\end{aligned}
$$


Since

$$
\operatorname{Res}_{s=1} \zeta(s, \alpha ; \mathfrak{a})=\frac{1}{q} \sum_{l=0}^{q-1} a_{l} \stackrel{\text { def }}{=} r
$$

we have

$$
\operatorname{Res}_{z=1-s} \frac{l_{n}(z, \alpha)}{z} \zeta(s+z, \alpha ; \mathfrak{a})=r \frac{l_{n}(1-s, \alpha)}{1-s} .
$$

By the definition,

$$
\rho\left(g_{1}, g_{2}\right)=\sum_{l=1}^{\infty} 2^{-l} \frac{\sup _{s \in K_{l}}\left|g_{1}(s)-g_{2}(s)\right|}{1+\sup _{s \in K_{l}}\left|g_{1}(s)-g_{2}(s)\right|}, \quad g_{1}, g_{2} \in H(D),
$$

where $\left\{K_{l}: l \in \mathbb{N}\right\} \subset D$ is a sequence of compact sets such that $D=\bigcup_{l=1}^{\infty} K_{l}$, $K_{l} \subset K_{l+1}$ for all $l \in \mathbb{N}$, and if $K \subset D$ is a compact set, then $K$ lies in some $K_{l}$. Therefore, it suffices to prove that, for every compact set $K$,

$$
\lim _{n \rightarrow \infty} \limsup _{T \rightarrow \infty} \frac{1}{T} \int_{0}^{T} \sup _{s \in K}\left|\zeta(s+i \tau, \alpha ; \mathfrak{a})-\zeta_{n}(s+i \tau, \alpha ; \mathfrak{a})\right| \mathrm{d} \tau=0 .
$$

Thus, let $K$ be a fixed compact subset of $D$. Suppose that $\varepsilon>0$ is such that $\frac{1}{2}+2 \varepsilon \leqslant \operatorname{Re} s \leqslant 1-\varepsilon$ for any point $s \in K$. Denote by $s=\sigma+i v$ the points of $K$. Moreover, let

$$
\beta=\sigma-\varepsilon-\frac{1}{2} \quad \text { and } \quad \theta=\frac{1}{2}+\varepsilon .
$$

Then, from (2.4) and (2.5) we find

$$
\begin{aligned}
\mid \zeta(s+ & i \tau, \alpha ; \mathfrak{a})-\zeta_{n}(s+i \tau, \alpha ; \mathfrak{a})\left|\leqslant \frac{1}{2 \pi} \int_{-\infty}^{\infty}\right| \zeta(s+i \tau-\beta+i t, \alpha ; \mathfrak{a}) \mid \\
& \times \frac{\left|l_{n}(-\beta+i t, \alpha)\right|}{|-\beta+i t|} \mathrm{d} t+|r| \frac{\left|l_{n}(1-s-i \tau, \alpha)\right|}{|1-s-i \tau|} .
\end{aligned}
$$

Recall that $s=\sigma+i v$. We take $t$ in place of $t+v$ in the above integral. This shift gives

$$
\begin{aligned}
& \left|\zeta(s+i \tau, \alpha ; \mathfrak{a})-\zeta_{n}(s+i \tau, \alpha ; \mathfrak{a})\right| \\
& \leqslant \frac{1}{2 \pi} \int_{-\infty}^{\infty}\left|\zeta\left(\frac{1}{2}+\varepsilon+i(t+\tau), \alpha ; \mathfrak{a}\right)\right| \frac{\left|l_{n}(1 / 2+\varepsilon-s+i t, \alpha)\right|}{|1 / 2+\varepsilon-s+i t|} \mathrm{d} t \\
& \quad+|r| \frac{\left|l_{n}(1-s-i \tau, \alpha)\right|}{|1-s-i \tau|} .
\end{aligned}
$$

From this, we obtain

$$
\frac{1}{T} \int_{0}^{T} \sup _{s \in K}\left|\zeta(s+i \tau, \alpha ; \mathfrak{a})-\zeta_{n}(s+i \tau, \alpha ; \mathfrak{a})\right| \mathrm{d} \tau \leqslant I_{1}+I_{2},
$$

where

$$
\begin{aligned}
I_{1}=\frac{1}{2 \pi} \int_{-\infty}^{\infty} & \left(\frac{1}{T} \int_{0}^{T}\left|\zeta\left(\frac{1}{2}+\varepsilon+i(t+\tau), \alpha ; \mathfrak{a}\right)\right| \mathrm{d} \tau\right) \\
& \times \sup _{s \in K} \frac{\left|l_{n}(1 / 2+\varepsilon-s+i t, \alpha)\right|}{|1 / 2+\varepsilon-s+i t|} \mathrm{d} t
\end{aligned}
$$


and

$$
I_{2}=|r| \frac{1}{T} \int_{0}^{T} \sup _{s \in K} \frac{\left|l_{n}(1-s-i \tau, \alpha)\right|}{|1-s-i \tau|} \mathrm{d} \tau .
$$

By the definition of $l_{n}(s, \alpha)$ and the estimate

$$
\Gamma(\sigma+i t) \ll \mathrm{e}^{-c|t|}, \quad c>0,
$$

uniform for $\sigma_{1} \leqslant \sigma \leqslant \sigma_{2}$, we find

$$
\begin{aligned}
\frac{\left|l_{n}(1 / 2+\varepsilon-s+i t, \alpha)\right|}{|1 / 2+\varepsilon-s+i t|} & \ll \frac{(n+\alpha)^{-\varepsilon}}{\theta} \exp \left\{-\frac{c}{\theta}|t-v|\right\} \\
& \ll{ }_{\theta, K}(n+\alpha)^{-\varepsilon} \exp \left\{-\frac{c}{\theta}|t|\right\} .
\end{aligned}
$$

It is well known that, for

$$
\int_{0}^{T}\left|\zeta\left(\frac{1}{2}+\varepsilon+i t, \alpha\right)\right|^{2} \mathrm{~d} t \ll T .
$$

Hence, in view of (1.1),

$$
\int_{0}^{T}\left|\zeta\left(\frac{1}{2}+\varepsilon+i t, \alpha ; \mathfrak{a}\right)\right|^{2} \mathrm{~d} t \ll T .
$$

Therefore,

$$
\begin{aligned}
\int_{0}^{T} & \left|\zeta\left(\frac{1}{2}+\varepsilon+i(t+\tau), \alpha ; \mathfrak{a}\right)\right| \mathrm{d} \tau \\
& \ll\left(T \int_{0}^{T}\left|\zeta\left(\frac{1}{2}+\varepsilon+i(t+\tau), \alpha ; \mathfrak{a}\right)\right|^{2} \mathrm{~d} \tau\right)^{1 / 2} \ll T(1+|t|) .
\end{aligned}
$$

This and (2.8) imply the estimate

$$
I_{1} \ll_{\theta, K}(n+\alpha)^{-\varepsilon} \int_{-\infty}^{\infty}(1+|t|) \exp \left\{-\frac{c}{\theta}|t|\right\} \mathrm{d} t \ll_{\theta, K}(n+\alpha)^{-\varepsilon} .
$$

Similarly as above, we obtain that

$$
\sup _{s \in K} \frac{\left|l_{n}(1-s-i \tau, \alpha)\right|}{|1-s-i \tau|} \ll_{\theta, K}(n+\alpha)^{1-\sigma} \exp \left\{-\frac{c}{\theta}|\tau|\right\} .
$$

Hence,

$$
I_{2} \ll_{\theta, K}|r| \frac{(n+\alpha)^{1 / 2-2 \varepsilon}}{T} .
$$

Now, (2.7), (2.9) and (2.10) imply the equality (2.6).

Now, we will examine the sequence $\left\{V_{n, \alpha, \mathfrak{a}}: n \in \mathbb{N}\right\}$, where $V_{n, \alpha, \mathfrak{a}}$ is the limit measure in Lemma 2. 
Lemma 4. The sequence $\left\{V_{n, \alpha, \mathfrak{a}}: n \in \mathbb{N}\right\}$ is relatively compact, i.e., every subsequence $\left\{V_{n_{k}, \alpha, \mathfrak{a}}\right\} \subset\left\{V_{n, \alpha, \mathfrak{a}}\right\}$ contains an another subsequence weakly convergent to a certain probability measure on $(H(D), \mathcal{B}(H(D)))$.

Proof. In virtue of the Prokhorov theorem [1, Theorem 6.1], it is sufficient to prove that the sequence $\left\{V_{n, \alpha, \mathfrak{a}}\right\}$ is tight, i.e., for every $\varepsilon>0$, there exists a compact set $K=K(\varepsilon) \subset H(D)$ such that

$$
V_{n, \alpha, \mathfrak{a}}(K)>1-\varepsilon
$$

for all $n \in \mathbb{N}$.

Let $\xi$ be a random variable on a certain probability space with measure $\mathbb{P}$ and uniformly distributed on $[0,1]$, and let $Y_{n, \alpha, \mathfrak{a}}=Y_{n, \alpha, \mathfrak{a}}(s)$ be the $H(D)$ valued random element whose distribution is $V_{n, \alpha, \mathfrak{a}}$. Moreover, let

$$
X_{T, n, \alpha, \mathfrak{a}}=X_{T, n, \alpha, \mathfrak{a}}(s)=\zeta_{n}(s+i T \xi, \alpha ; \mathfrak{a}) .
$$

Then the assertion of Lemma 2 is equivalent to the relation

$$
X_{T, n, \alpha, \mathfrak{a}} \underset{T \rightarrow \infty}{\stackrel{\mathcal{D}}{\longrightarrow}} Y_{n, \alpha, \mathfrak{a}}
$$

We have mentioned that the series for $\zeta_{n}(s, \alpha ; \mathfrak{a})$ is absolutely convergent for $\sigma>\frac{1}{2}$. Hence, by properties of absolutely convergent series, for fixed $\frac{1}{2}<\sigma<1$,

$$
\begin{aligned}
\lim _{T \rightarrow \infty} \frac{1}{T} \int_{0}^{T}\left|\zeta_{n}(\sigma+i t, \alpha ; \mathfrak{a})\right|^{2} \mathrm{~d} t & =\sum_{m=0}^{\infty} \frac{\left|a_{m}\right|^{2} v_{n}^{2}(m, \alpha)}{(m+\alpha)^{2 \sigma}} \\
& \leqslant \sum_{m=0}^{\infty} \frac{\left|a_{m}\right|^{2}}{(m+\alpha)^{2 \sigma}} \leqslant C_{\alpha, \mathfrak{a}}<\infty .
\end{aligned}
$$

Let $K_{l}$ be a compact set from the definition of the metric $\rho$. Then (2.12) and the Cauchy integral formula imply

$$
\sup _{n \in \mathbb{N}} \limsup _{T \rightarrow \infty} \frac{1}{T} \int_{0}^{T} \sup _{s \in K_{l}}\left|\zeta_{n}(s+i \tau, \alpha ; \mathfrak{a})\right| \mathrm{d} \tau \leqslant R_{l, \alpha, \mathfrak{a}}<\infty .
$$

Now, taking $M=M_{l, \alpha, \mathfrak{a}}(\varepsilon)=R_{l, \alpha, \mathfrak{a}} 2^{l} \varepsilon^{-1}$, where $\varepsilon>0$ is an arbitrary fixed number, we find that

$$
\begin{aligned}
\limsup _{T \rightarrow \infty} \mathbb{P} & \left(\sup _{s \in K_{l}}\left|X_{T, n, \alpha, \mathfrak{a}}(s)\right|>M\right) \\
& =\limsup _{T \rightarrow \infty} \frac{1}{T} \operatorname{meas}\left\{\tau \in[0, T]: \sup _{s \in K_{l}}\left|\zeta_{n}(s+i \tau, \alpha ; \mathfrak{a})\right|>M\right\} \\
& \leqslant \sup _{n \in \mathbb{N}} \limsup _{T \rightarrow \infty} \frac{1}{T M} \int_{0}^{T} \sup _{s \in K_{l}}\left|\zeta_{n}(s+i \tau, \alpha ; \mathfrak{a})\right| \mathrm{d} \tau \leqslant \frac{\varepsilon}{2^{l}}
\end{aligned}
$$

for all $n$ and $l \in \mathbb{N}$. The set

$$
K=K(\varepsilon)=\left\{g \in H(D): \sup _{s \in K_{l}}|g(s)| \leqslant M_{l, \alpha, \mathfrak{a}}(\varepsilon), l \in \mathbb{N}\right\}
$$


is compact in the space $H(D)$, and, by (2.11) and (2.13),

$$
\mathbb{P}\left(Y_{n, \alpha, \mathfrak{a}} \in K\right) \geqslant 1-\varepsilon
$$

for all $n \in \mathbb{N}$. Hence,

$$
V_{n, \alpha, \mathfrak{a}}(K) \geqslant 1-\varepsilon
$$

for all $n \in \mathbb{N}$.

Now, we are in position to prove Theorem 7 .

Proof. In view of Lemma 4, there exists a subsequence $\left\{V_{n_{k}, \alpha, \mathfrak{a}}\right\} \subset\left\{V_{n, \alpha, \mathfrak{a}}\right\}$ weakly convergent to a certain probability measure $P_{\alpha, \mathfrak{a}}$ on $(H(D), \mathcal{B}(H(D)))$ as $k \rightarrow \infty$. Thus, we have the relation

$$
Y_{n_{k}, \alpha, \mathfrak{a}} \underset{k \rightarrow \infty}{\stackrel{\mathcal{D}}{\longrightarrow}} P_{\alpha, \mathfrak{a}} .
$$

Define

$$
X_{T, \alpha, \mathfrak{a}}=X_{T, \alpha, \mathfrak{a}}(s)=\zeta(s+i T \xi, \alpha ; \mathfrak{a}) .
$$

Then an application of Lemma 3 gives, for every $\varepsilon>0$,

$$
\begin{aligned}
& \lim _{n \rightarrow \infty} \limsup _{T \rightarrow \infty} \mathbb{P}\left(\rho\left(X_{T, \alpha, \mathfrak{a}}, X_{T, n, \alpha, \mathfrak{a}}\right) \geqslant \varepsilon\right) \\
& =\lim _{n \rightarrow \infty} \limsup _{T \rightarrow \infty} \frac{1}{T} \text { meas }\left\{\tau \in[0, T]: \rho\left(\zeta(s+i \tau, \alpha ; \mathfrak{a}), \zeta_{n}(s+i \tau, \alpha ; \mathfrak{a})\right) \geqslant \varepsilon\right\} \\
& \leqslant \lim _{n \rightarrow \infty} \limsup _{T \rightarrow \infty} \frac{1}{T \varepsilon} \int_{0}^{T} \rho\left(\zeta(s+i \tau, \alpha ; \mathfrak{a}), \zeta_{n}(s+i \tau, \alpha ; \mathfrak{a})\right) \mathrm{d} \tau=0 .
\end{aligned}
$$

This equality, and relations (2.11) and (2.14) show that the hypotheses of Theorem 4.2 of [1] are satisfied. Hence,

$$
X_{T, \alpha, \mathfrak{a}} \underset{T \rightarrow \infty}{\stackrel{\mathcal{D}}{\longrightarrow}} P_{\alpha, \mathfrak{a}},
$$

or equivalently, $P_{T, \alpha, \mathfrak{a}}$ converges weakly to $P_{\alpha, \mathfrak{a}}$ as $T \rightarrow \infty$.

Corollary 1. Suppose that $\Phi: H(D) \rightarrow H(D)$ is a continuous operator, and, for $A \in \mathcal{B}(H(D))$,

$$
P_{T, \Phi, \alpha, \mathfrak{a}}(A)=\frac{1}{T} \operatorname{meas}\{\tau \in[0, T]: \Phi(\zeta(s+i \tau, \alpha ; \mathfrak{a})) \in A\} .
$$

Then $P_{T, \Phi, \alpha, \mathfrak{a}}$ converges weakly to $P_{\alpha, \mathfrak{a}} \Phi^{-1}$ as $T \rightarrow \infty$.

Proof. It follows from the definitions of $P_{T, \Phi, \alpha, \mathfrak{a}}$ and $P_{T, \alpha, \mathfrak{a}}$ that $P_{T, \Phi, \alpha, \mathfrak{a}}=$ $P_{T, \alpha, \mathfrak{a}} \Phi^{-1}$. Thus, the corollary is a result of Theorem 7 , the continuity of $\Phi$ and Theorem 5.1 of [1]. 


\section{Proof of the main theorems}

Proof. (Proof of Theorem 3). Let $F_{\alpha, \mathfrak{a}}$ be the support of the limit measure $P_{\alpha, \mathfrak{a}}$ in Theorem 7. By the definition of a support, $F_{\alpha, \mathfrak{a}}$ is a minimal closed set of the space $H(D)$ such that $P_{\alpha, \mathfrak{a}}\left(F_{\alpha, \mathfrak{a}}\right)=1$. Thus, $F_{\alpha, \mathfrak{a}} \neq \varnothing$, and consists of all elements $g \in H(D)$ such that, for every open neighbourhood $G$ of $g$, the inequality $P_{\alpha, \mathfrak{a}}(G)>0$ is satisfied.

For $f \in F_{\alpha, \mathfrak{a}}$, define the set

$$
G_{\varepsilon}=\left\{g \in H(D): \sup _{s \in K}|g(s)-f(s)|<\varepsilon\right\} .
$$

Then $G_{\varepsilon}$ is an open neighbourhood of the element $f$ of the support of the measure $P_{\alpha, \mathfrak{a}}$. Therefore $P_{\alpha, \mathfrak{a}}\left(G_{\varepsilon}\right)>0$. Now, using the equivalent of weak convergence of probability measures in terms of open sets [1, Theorem 2.1], we deduce from Theorem 7 the inequality

$$
\liminf _{T \rightarrow \infty} P_{T, \alpha, \mathfrak{a}}\left(G_{\varepsilon}\right) \geqslant P_{\alpha, \mathfrak{a}}\left(G_{\varepsilon}\right)>0 .
$$

This and the definitions of $P_{T, \alpha, \mathfrak{a}}$ and $G_{\varepsilon}$ prove the theorem.

Proof. (Proof of Theorem 4). We preserve the notation of the set $G_{\varepsilon}$. The boundary $\partial G_{\varepsilon}$ of $G_{\varepsilon}$ lies in the set $\left\{g \in H(D): \sup _{s \in K}|g(s)-f(s)|=\varepsilon\right\}$. Hence, we have that $\partial G_{\varepsilon_{1}} \cap \partial G_{\varepsilon_{2}}=\varnothing$ for $\varepsilon_{1} \neq \varepsilon_{2}, \varepsilon_{1}>0, \varepsilon_{2}>0$. This shows that the set $G_{\varepsilon}$ is a continuity set of the measure $P_{\alpha, \mathfrak{a}}$, i.e., $P_{\alpha, \mathfrak{a}}\left(\partial G_{\varepsilon}\right)=0$, for all but at most countably many $\varepsilon>0$. Applying the equivalent of weak convergence of probability measures in terms of continuity sets [1, Theorem 2.1], we deduce from Theorem 7 the inequality

$$
\lim _{T \rightarrow \infty} P_{T, \alpha, \mathfrak{a}}\left(G_{\varepsilon}\right)=P_{\alpha, \mathfrak{a}}\left(G_{\varepsilon}\right)>0
$$

for all but at most countably many $\varepsilon>0$, and the definitions of $P_{T, \alpha, \mathfrak{a}}$ and $G_{\varepsilon}$ prove the theorem.

Proof. (Proof of Theorem 5). By Corollary 1, $P_{T, \Phi, \alpha, \mathfrak{a}}$ converges weakly to $P_{\alpha, \mathfrak{a}} \Phi^{-1}$ as $T \rightarrow \infty$. We will show that the support of the measure $P_{\alpha, \mathfrak{a}} \Phi^{-1}$ contains the closure of the set $\Phi\left(F_{\alpha, \mathfrak{a}}\right)$.

We suppose that, for every open set $\hat{G} \neq \varnothing$, the set $\left(\Phi^{-1} \hat{G}\right) \cap F_{\alpha, \mathfrak{a}}$ is non-empty. Let $g$ be an arbitrary element of the set $\Phi\left(F_{\alpha, \mathfrak{a}}\right)$, and $G$ be its any open neighbourhood. Then, the set $\Phi^{-1} G$ is open, and contains an element of the set $F_{\alpha, \mathfrak{a}}$. However, $F_{\alpha, \mathfrak{a}}$ is the support of the measure $P_{\alpha, \mathfrak{a}}$. Therefore, $P_{\alpha, \mathfrak{a}}\left(\Phi^{-1} G\right)>0$, and

$$
P_{\alpha, \mathfrak{a}} \Phi^{-1}(G)=P_{\alpha, \mathfrak{a}}\left(\Phi^{-1} G\right)>0 .
$$

Hence, the support of the measure $P_{\alpha, \mathfrak{a}} \Phi^{-1}$ contains the set $\Phi\left(F_{\alpha, \mathfrak{a}}\right)$, and, as a closed set, contains the closure of $\Phi\left(F_{\alpha, \mathfrak{a}}\right)$.

Now, suppose that, for every polynomial $p=p(s)$, the set $\left(\Phi^{-1}\{p\}\right) \cap F_{\alpha, \mathfrak{a}}$ is non-empty. We will show that then, for every open set $G \subset H(D)$, the 
set $\left(\Phi^{-1} G\right) \cap F_{\alpha, \mathfrak{a}}$ is non-empty. It is well known that the approximation in the space $H(D)$ reduces to that on compact sets with connected complements, i.e., in the definition of the metric $\rho$ we may take the sets $K_{l}$ with connected complements. Let $K \subset D$ be a compact set with connected complement, and $g \in G$. Then, by the Mergelyan theorem [7], for every $\varepsilon>0$ there exists a polynomial $p=p(s)$ such that

$$
\sup _{s \in K}|g(s)-p(s)|<\frac{\varepsilon}{2} .
$$

Then, by the above remark, we have that $\rho(g, p)<\varepsilon$ provided the set $K$ is well chosen. Thus, if $\varepsilon$ is small enough, then $p \in G$. Therefore, $\Phi^{-1}\{p\} \subset \Phi^{-1} G$, and the set $\left(\Phi^{-1} G\right) \cap F_{\alpha, \mathfrak{a}} \neq \varnothing$ if $\left(\Phi^{-1}\{p\}\right) \cap F_{\alpha, \mathfrak{a}} \neq \varnothing$.

For $f \in \Phi\left(F_{\alpha, \mathfrak{a}}\right)$, define the set

$$
G_{\varepsilon}=\left\{g \in H(D): \sup _{s \in K}|g(s)-f(s)|<\varepsilon\right\} .
$$

Then $G_{\varepsilon}$ is an open neighbourhood of the element $f$ of the support of the measure $P_{\alpha, \mathfrak{a}} \Phi^{-1}$. Thus $P_{\alpha, \mathfrak{a}} \Phi^{-1}\left(G_{\varepsilon}\right)>0$. Hence, by Corollary 1 ,

$$
\liminf _{T \rightarrow \infty} P_{T, \Phi, \alpha, \mathfrak{a}}\left(G_{\varepsilon}\right) \geqslant P_{\alpha, \mathfrak{a}} \Phi^{-1}\left(G_{\varepsilon}\right)>0 .
$$

This and the definitions of $P_{T, \Phi, \alpha, \mathfrak{a}}$ and $G_{\varepsilon}$ give the assertion of the theorem.

Proof. (Proof of Theorem 6) We repeat with evident changes the arguments of the proof of Theorem 4 .

\section{Acknowledgements}

The research of the second author is funded by the European Social Fund according to the activity "Improvement of researchers" qualification by implementing world-class R\&D projects' of Measure No. 09.3.3-LMT-K-712-010037.

\section{References}

[1] P. Billingsley. Convergence of Probability Measures. Willey, New York, 1968.

[2] A. Javtokas and A. Laurinčikas. On the periodic Hurwitz zeta-function. HardyRamanujan J., 29:18-36, 2006.

[3] A. Javtokas and A. Laurinčikas. Universality of the periodic Hurwitz zetafunction. Integral Transforms Spec. Funct., 17:711-722, 2006.

[4] A. Laurinčikas. Limit Theorems for the Riemann Zeta-Function. Kluwer, Dordrecht, 1996.

[5] A. Laurinčikas, R. Macaitienè, D. Mochov and D. Šiaučiūnas. Universality of the periodic Hurwitz zeta-function with rational parameter. Sib. Math. J., 59(5):894900, 2018. 
[6] K. Matsumoto. A survey on the theory of universality for zeta and $L$-functions. In M. Kaneko, S. Kanemitsu and J. Liu(Eds.), Number Theory: Plowing and Starring Through High Wawe Forms, Proc. 7th China-Japan Semin. (Fukuoka 2013), volume 11 of Number Theory and Appl., pp. 95-144, New Jersey, London, Singapore, Beijing, Shanghai, Hong Kong, Taipei, Chennai, 2015. World Scientific Publishing Co.

[7] S.N. Mergelyan. Uniform approximations to functions of complex variable. Usp. Mat. Nauk., 7:31-122, 1952 (in Russian).

[8] J. Steuding. Value-Distribution of L-Functions. Lecture Notes Math. vol. 1877, Springer, Berlin, Heidelberg, 2007.

[9] S.M. Voronin. Theorem on the "universality" of the Riemann zeta-function. Izv. Akad. Nauk SSSR, Ser. Matem., 39:475-486, 1975 (in Russian). 